\title{
Plakophilin 4 and ARVCF expression in a bullous cutaneous drug reaction
}

\author{
Ana Maria Abreu Velez¹, David J. Cohen², Michael S. Howard ${ }^{1}$ \\ ${ }^{1}$ Georgia Dermatopathology Associates, Atlanta, Georgia USA, ${ }^{2}$ Dermatologic Surgery Specialists, PC, Macon, Georgia, USA
}

Corresponding author: Ana Maria Abreu Velez, E-mail: abreuvelez@yahoo.com

\begin{abstract}
The subepidermal vesiculobullous disorders include a wide variety of pathogenically unrelated entities, which share the formation of clefts or bullae. A 59 year old female presented with a sudden eruption of pruritic skin vesicles and blisters on several areas of her body. The patient was taking multiple medications. We decided to test for the expression of p0071 and ARVCF because they are linked with tight, adherens, and occludens cell junctions in the epidermis and the dermis, and study if these molecules participate in the bullae formation. Skin biopsies were taken from lesional skin and were tested by hematoxylin and eosin(H\&E) staining, as well as via immunohistochemistry (IHC) and direct immunofluorescence(DIF) using multiple antibodies. ARVCF and p0071 were overexpressed in the epidermis, and in dermal cell junctions around the blisters along with ribosomal protein S6-pS240, Factor XIIIa, CD15, CD45, multiple immunoglobulins, complement, fibrinogen and HLA-DP, DQ, DR antigen. All these molecules were also overexpressed around dermal vessels, eccrine glands and in neurovascular cell junctions below the blister. A normal control did not display the overexpression. Drug reactions may cause blisters; regional cell junctions may be altered, as demonstrated by overexpression of ARVCF and p0071. The overexpression likely contributes to passage of immunologic cells and formation of edema, directly contributing to blister formation.
\end{abstract}

Key words: Subepidermal blisters; Drug reactions; Plakophilin 4; ARVCF

\section{INTRODUCTION}

Drug eruptions often present with blisters [1,2]. Sometimes these blisters present when patients are taking multiple medications. Little is known about cell junction alterations and their putative roles in drug reactions. Here we described an interesting case, suggesting involvement of altered cell junctions in blister formation.

\section{CASE REPORT}

Here we present a 59 year old female, who presented with a sudden eruption of pruritic skin vesicles and blisters. The patient was taking NitroQuick ${ }^{\circledR}$ $0.4 \mathrm{mgs} /$ day, Phentanyl $75^{\circledR} \mathrm{mg} /$ day, ProAir HFA $90^{\circledR}$ mgs/day, Tetracycline $500 \mathrm{mg} /$ day, Vectical ${ }^{\circledR} 3$ mcg and Pamelor ${ }^{\circledR} 50 \mathrm{mg} /$ day (for medical depression and some breathing difficulties). Skin biopsies were taken for hematoxylin and eosin (H\&E) staining, for direct immunofluorescence (DIF) and for immunohistochemical (IHC) staining; their processing was performed as previously described [2]. The pathology results demonstrated a subepidermal blister due to a drug reaction, and the patient was evaluated to determine which medication was causing the reaction. The patient was provided with Phenergan ${ }^{\circledR 5}$ mgs/day, and Olux $\mathrm{E}^{\circledR}$ topical foam with improvement.

We utilized normal skin samples from breast reduction surgery as controls. For the DIF, we utilized standard panel antibodies as previously described [2]. In addition to these, we utilized a polyclonal antibody to Armadillo Repeat Gene Deleted in Velo-Cardio-Facial syndrome (ARVCF) (source guinea pig and tested in human and bovine, Catalog No. GP155, dilution 1:20; from Progen Biotechnik, Heidelberg, Germany); as its secondary, we utilized goat anti-guinea pig $\operatorname{IgG}(\mathrm{H} \& \mathrm{~L})$, conjugated

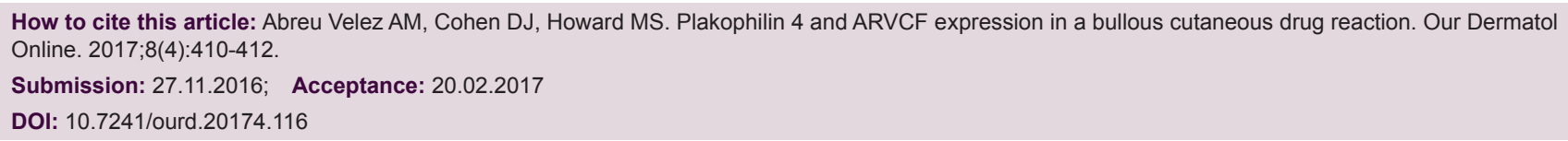


with Alexa 555 (2 mg/ml; 0.5ml) from Invitrogen (Carlsbad, California, USA), Catalog No. A.21435. We also utilized anti-p007l (mouse monoclonal multi-epitope cocktail, Catalog No. 641166) from Progen and as its secondary, a Texas red conjugated goat anti-mouse $\operatorname{IgG}$, at a 1:50 dilution (Invitrogen, Catalog No. T862).

We further utilized polyclonal rabbit anti-human myeloperoxidase, monoclonal mouse anti-human antibodies to cyclo-oxygenase 2 (COX-2), anti-human HLA-DP, DQ, DR antigen, CD4, CD8, CD15, CD45; ribosomal protein S6-pS240/phosphorylation site specific (Ribo), and Factor XIIIA(all from Novocastra/Leica, Buffalo Grove, Illinois, USA).

The H\&E staining demonstrated a subepidermal blister, with partial re-epithelialization of the blister base. Within the blister lumen, no significant cellular infiltrate was seen (Fig. la, red arrow, 100X). Dermal papillary festoons were observed; within the dermis, a mild, superficial, perivascular infiltrate of

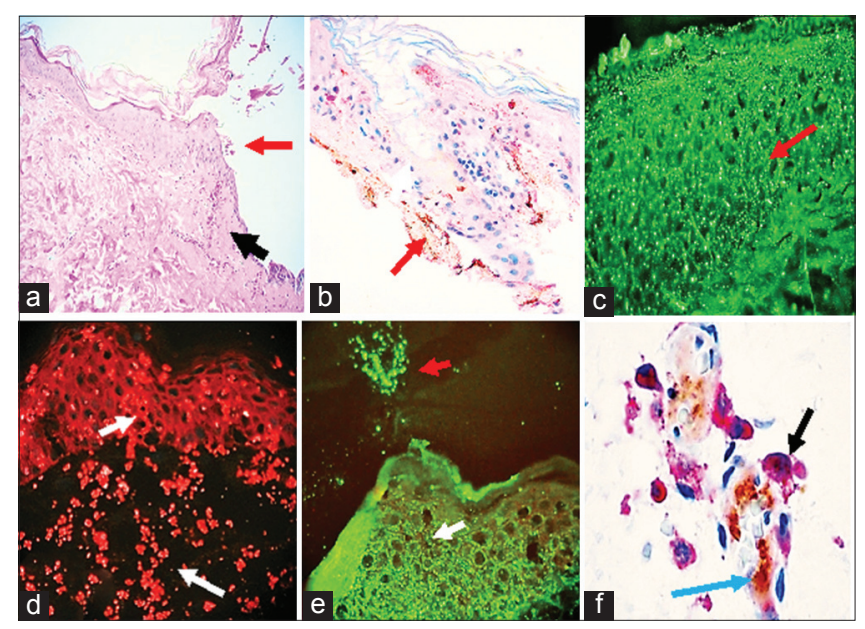

Figure 1: (a) H\&E staining shows a subepidermal blister base(red arrow); the black arrow shows the lymphohistiocytic infiltrate around upper dermal vessels (100X). (b) Double IHC staining, showing positive staining for CD15 (brown staining) and COX-2(red staining) near the epidermis (red arrow). (c)DIF using FITC conjugated anti-human fibrinogen antibodies shows positive dot pattern staining in several epidermal cell junctions (antigens of unknown nature; yellow staining; red arrow). (d) DIF showing overexpression of p0071 in the neurovascular net around eccrine gland ducts. Notably, p0071 seems to be reactive to material extruded though the eccrine ducts. The epidermis also showed positive staining for p0071 (red staining; white arrows). (e) DIF using FITC conjugated anti-human fibrinogen antibody, and showing a detail of the reactive material extruded through an eccrine gland acrosyringium (green/yellow staining; red arrow). The white arrow highlights reactivity on epidermal cell junctions. (f) Double IHC staining, showing positivity around upper dermal blood vessels for Factor XIIla (brown staining; blue arrow), in proximity to CD45 (red staining; black arrow). lymphocytes, histiocytes, neutrophils and occasional eosinophils was identified. DIF displayed $\operatorname{IgG}(+++$, on focal cell junctions in the epidermis and dermis; no classic intercellular staining between epidermal keratinocytes was seen); $\operatorname{IgE}$ ( + , focal dermal positivity); Complement/Clq (+, linear at the dermal/epidermal junction and on c-ANCAS); Complement/C3 (+, focal linear at the base membrane zone (BMZ), and focally on dermal neural structures); Lambda light chains $(+$, focal cell junctions in the dermis); albumin $(+++$, focal cell junctions in the epidermis and dermis) and fibrinogen $(+++$, neurovascular structures). ARVCF was overexpressed throughout the dermis $(++++)$; p0071 was overexpressed in the epidermis ++++ ), as well as on blood vessels near eccrine glands and on material excreted through eccrine glands $(++++)$. Our normal skin controls displayed p007l (+), and ARVCF (+), stains. The other immunoglobulin, complement and inflammatory cell markers were negative.

Our IHC results demonstrated strong reactivity with Factor XIIIa in cells around the dermal blood vessels. HLA-DP, DQ, DR antigen was also very positive around these vessels (Fig. 1). The inflammatory infiltrate around the vessels was also positive for CD45; this staining highlighted fragmented lymphocytes. Both CD4 and CD68 were negative, and only few cells were positive for CD8 and COX-2 around the dermal blood vessels. Ribo was positive on epidermal cells surrounding the blisters. Some CD15 positive cells were noted around the blisters, and around upper dermal blood vessels.

\section{DISCUSSION}

Blistering drug eruptions are common, possibly due to an aging population and the increased number of medications that many patients are utilizing; the overall drug reaction patterns seem to be more complex than previously described [1-2]. Many drug reactions may involve excretions of drug breakdown products through the sweat glands, as shown in this case via overexpression of p0071, ARVCF and other markers [3]. Here, we detected reactivity with secondary intracellular cell signaling molecules (Ribo), as well as immunoglobulins, complement, and CD15 to epidermal and dermal antigens. Notably, p0071 is a plakophilin and a member of the p120 (ctn) family of armadillo-related proteins [4]. Here we demonstrated overexpression of p0071 and ARVCF; 
these molecules are linked with tight, adherens, and occludens cell junctions. We hypothesize that edema and inflammatory cells penetrate the blister through altered cell junctions [3] ARVCF and p0071 molecules are also present in in the cells junctions of the vessels and nerves.

We speculate that the drug reaction elicits inflammation, facilitating the passage of cells as well as serum from adjacent vessels that are overexpressing HLA-DR,DP,DQ antigen. We speculate that additional cells, present in the dermis before initiation of the drug reaction are also involved. In our case, such a constellation of events and cells staining positive for Factor XIIIa, CD15 and CD45 were likely involved in the pathologic process as well as some drug metabolites excreting through the acrosyringia of eccrine glands.

Excretion of material by eccrine glands has previously been described by others in drug reactions [3]. We have previously described increased expression of p0071 in another drug reaction [2]; we speculate that both p0071 and ARVCF not only play roles in maintaining tissue integrity and cell adhesion, but also have putative roles in cell signaling and may help inflammatory mediators to move through cell junctions, as shown by the overexpression of these two molecules. We noted a CD8 T cell response and intensification of HLA-DP, $\mathrm{DQ}, \mathrm{DR}$ antigen, COX-2 and Ribo expression in the lesion, suggesting not only a non-specific immune response(COX-2), but also a more specific immune response with antigen presentation through the HLADP,DQ,DR and T cell interactions; secondary cell signaling is likely occurring via Ribo. These features may explain why some people who have experienced one drug reaction are prone to present with additional drug reactions (possibly due to an immune memory response). In our case ARVCF was overexpressed throughout the dermis. It is known that ARVCF binds to the PDZ-domain of zonula occludens ZO-1 and ZO-2 tight junction proteins [5].

Our findings may explain the dot-like reactivity seen in both the dermis and the epidermis with p0071 and ARVCF as a response against likely cell junctions, suggesting that, at least in this case, the drugs acted as triggering factor(s). Specifically, epitopes on cells junctions and/or other molecules were presented as putative antigens through the HLA-DP,DQ,DR overexpression. p0071 has been described as a protein with dual localization in adherens junctions and desmosomes, depending on the cell type examined; here we clearly see an extrusion of p007l (possibly part of damaged adherens junctions, extruded by the immune response through eccrine sweat glands; see Fig. 1).

In summary, we observed strong reactivity to epidermal and dermal cell junctions in a drug reaction via DIF and IHC. Drug reactions may cause blisters; here, we demonstrated cell junction marker expression for p0071/plakophilin and ARVCF. The overexpression of these markers may in turn contribute to passage of immunologic cells and edema in blister formation, as well as drug metabolite excretion through acrosyringia of eccrine glands. Few publications in the medical literature have described $\mathrm{p} 007 \mathrm{l}$ and ARVCF expression in cutaneous drug reactions.

\section{Abbreviations}

Basement membrane zone (BMZ), direct immunofluorescence (DIF), immunohistochemistry (IHC), hematoxylin and eosin staining (H\&E), armadillo repeat gene deleted in velo-cardiofacial syndrome (ARVCF), tight junctions (TJs), adherens junctions (AJs), ribosomal protein S6-pS240/phosphorylation site specific (Ribo), cyclo-oxygenase 2 (COX-2), 4',6-diamidino-2phenylindole (DAPI).

\section{REFERENCES}

1. Cassandra M, Morgan M. The intraepidermal blistering conditions. Semin Cutan Med Surg. 2004;23:2-9.

2. Abreu Velez AM, Barth G, Howard MS. Thrombomodulin overexpression surrounding a subepidermal bullous allergic drug reaction. Our Dermatol Online. 2013;4:514-6.

3. Johnson HL, Maibach HI. Drug excretion in human eccrine sweat. J Invest Dermatol. 1971;56:182-8.

4. Hatzfeld M. Plakophilins: Multifunctional proteins or just regulators of desmosomal adhesion? Biochim Biophys Acta. 2007;1773:69-7.

5. Kausalya PJ, Phua DCY, Hunziker W. Association of ARVCF with zonula occludens (ZO)-1 and ZO-2: Binding to PDZ-Domain proteins and cell-cell adhesion regulate plasma membrane and nuclear localization of ARVCF. Mol Biol Cell. 2004;15:5503-5.

Copyright by Ana Maria Abreu Velez, et al. This is an open access article distributed under the terms of the Creative Commons Attribution License, which permits unrestricted use, distribution, and reproduction in any medium, provided the original author and source are credited.

Source of Support: Georgia Dermatopathology Associates, Atlanta, Georgia, USA, Conflict of Interest: None declared. 